\title{
Magnetic behavior of sputtered Co-doped indium-tin oxide films
}

\author{
Jolanta Stankiewicz, ${ }^{1, *}$ Francisco Villuendas, ${ }^{1}$ and Juan Bartolomé ${ }^{1}$ \\ ${ }^{1}$ Instituto de Ciencia de Materiales de Aragón Consejo Superior de Investigaciones Científicas and Universidad de Zaragoza, \\ 50009-Zaragoza, Spain \\ ${ }^{2}$ Departamento de Física Aplicada, Universidad de Zaragoza, 50009-Zaragoza, Spain
}

(Received 20 October 2006; revised manuscript received 28 March 2007; published 6 June 2007)

\begin{abstract}
We have studied Co-doped indium-tin oxide (ITO) films deposited by magnetron sputtering on fused-quartz substrates under various conditions. We find that the magnetic and electrical transport properties of these films vary strongly with post-growth treatment. In films with more than 8 at. \% of Co, we observe irreversible magnetization and an anomalous Hall effect that are likely caused by a mixed magnetic state, with metal-rich nanoclusters within a ITO:Co matrix. Homogeneous films with less than 8 at. $\%$ of Co are ferromagnetic at room temperature. Their magnetic behavior is consistent with a bound magnetic polaron percolation model.

DOI: $10.1103 /$ PhysRevB.75.235308

PACS number(s): 75.25.+z, 75.50.Pp, 81.05.Hd
\end{abstract}

\section{INTRODUCTION}

Diluted magnetic semiconductor (DMS) oxide systems that show a high-temperature ferromagnetic (FM) behavior have a great potential in the development of thin-film optoelectronic spin-based devices. ${ }^{1}$ In recent years, hightemperature FM behavior has been reported for $\mathrm{ZnO}$ films doped with $\mathrm{Mn},{ }^{2}$ for Co- and Fe-doped $\mathrm{TiO}_{2}$ films, ${ }^{3,4}$ and for indium-tin oxide (ITO) films doped with $\mathrm{Mn}, \mathrm{Ni}$, and $\mathrm{Cr}^{5-8}$ There is yet no consensus on how ferromagnetism comes about in DMS oxides. It is clear that growth processes and post-growth annealing strongly affect their properties. Particularly, metallic Co clusters are observed as often as homogeneous distributions of $\mathrm{Co}$ atoms in thin films. The origin of ferromagnetism in cobalt-doped films remains unclear even in the most extensively studied system, $\mathrm{TiO}_{2}$ : Co. Nearly all published papers on this system report similar magnetic and transport results. However, the interpretations are often controversial. In some cases, high-temperature annealing of epitaxial $\mathrm{TiO}_{2}$ : Co films grown by pulsed laser deposition seems to remove the metallic Co nanoclusters, existing in as-grown films, and to incorporate magnetic ions into the host matrix, which leads to intrinsic ferromagnetism. ${ }^{9}$ On the other hand, it has more recently been found that annealing, while removing metallic Co nanoclusters from the bulk, promotes their diffusion to the interface between substrate and film in the same system. ${ }^{10}$ Consequently, the observed ferromagnetism in annealed films arises from Co particles. It is clear that careful studies that involve and relate microstructural, magnetic, and electrical transport characterizations are required in order to establish the physical mechanism underlying magnetism in the oxide semiconductors. In addition, some high magnetic moment values found in DMS oxides are unprecedented and cannot be accounted for by any known ferromagnetic phase. ${ }^{11}$ Understanding magnetism in these materials is a great challenge for basic physics as well as for device applications.

In this paper, we report the observation of roomtemperature (RT) ferromagnetism in Co-doped ITO thin films. We aim to contribute to the understanding of magnetism in DMS oxides by relating results of structural, electrical transport, and magnetic studies of these films. ITO films with up to 8 at. \% of Co show an intrinsic FM behavior which correlates with their transport properties. On the other hand, a mixed magnetic state, likely with Co-rich clusters, seems to lead to the behavior that is encountered in films with larger Co content.

\section{EXPERIMENT}

We grew Co-doped ITO thin films by dc magnetron cosputtering from cobalt and ceramic ITO $\left(90 \% \operatorname{In}_{2} \mathrm{O}_{3}\right.$ and $10 \% \mathrm{SnO}_{2}$ by weight) targets mounted on two separate guns, in a vacuum system with a base pressure of $\sim 4 \times 10^{-6}$ Torr. The Sn concentration in the ITO target was constant. In each process, three pieces of fused-quartz substrates were placed on a heating block at different locations. In this way, we could obtain three films, each with a different Co content. The Sn and In content in films varied, depending on Co concentration, while the $\mathrm{Sn} / \mathrm{In}$ ratio remained constant. The partial pressure of argon was fixed at $1.1 \times 10^{-3}$ Torr during deposition. The substrate temperature was also kept constant at $200{ }^{\circ} \mathrm{C}$. The typical deposition rates were $0.5-1 \AA / \mathrm{s}$, and film thickness ranged from 400 to $800 \mathrm{~nm}$. After growth, some films were annealed in oxygen or air at various temperatures from 300 to $700{ }^{\circ} \mathrm{C}$, usually for $3 \mathrm{~h}$. We also used reductive annealing in Ar: $4 \% \mathrm{H}_{2}$ in several cases. We estimated the Co content and its distribution in the films from $\mathrm{x}$-ray photoelectron spectroscopy (XPS) and from electron probe microanalysis. XPS spectra, obtained using $\mathrm{Al} K \alpha$ radiation, also revealed the electronic state of Co ions. To this end, we etched the films' surface with argon ions. The rate of etching was approximately $0.5 \mathrm{~nm} / \mathrm{min}$. This procedure was repeated several times. After each etching cycle, Co $2 p$, In $3 d$, Sn $3 d$, and O $1 s$ spectra were recorded. The structural characterization of films was done with $\mathrm{x}$-ray diffraction (XRD) and transmission electron microscopy (TEM). A superconducting quantum interference device (SQUID) magnetometer was used for magnetic measurements. Since magnetic signals from thin films are quite small, we implement a modulation method in our measurements. The diamagnetic contribution from the substrate, which is linear in the magnetic field and does not vary with temperature, is subtracted from all data. This contributes significantly to the absolute 
error. The relative error obtained for magnetic measurements is about 1\%; absolute values are determined to within $20 \%$. Here van der Pauw geometry was used to obtain the resistivity and Hall effect data in magnetic fields of up to $5 \mathrm{~T}$. The Co content in the films varied between 2 and 15 at. \%.

\section{RESULTS AND DISCUSSION}

Indium-tin oxide is a transparent direct band-gap semiconductor. It crystallizes in a cubic bixbyite structure with a lattice parameter of $10.118 \AA$. Similar to its parent indium oxide, ITO is generally nonstoichiometric with respect to oxygen. It shows a rather high electrical conductivity, arising from a large electron concentration, which follows from doping with $\mathrm{Sn}$ and from the presence of oxygen vacancies. Since it exhibits technologically important properties, ITO is a widely studied material. ${ }^{12}$

The appearance of the as-deposited ITO:Co varies from a smoky-brown for Co-rich films to a pale-yellowish for films with a smaller Co content. The as-grown films with Co content higher than 10 at. \% are partially amorphous, weakly paramagnetic, and rather resistive (their RT resistivity is of $\sim 0.1 \Omega \mathrm{cm}$ ). Upon annealing, the films' color gradually disappears and the films change to a polycrystalline phase. Their conductivity first increases (up to $\sim 1 \times 10^{3} \Omega^{-1} \mathrm{~cm}^{-1}$ ), with increasing annealing temperature up to $400{ }^{\circ} \mathrm{C}$, but more resistive materials obtain at higher temperatures. On the other hand, the RT conductivity of the films with smaller Co concentration, which is $\sim(0.2-1) \times 10^{3} \Omega^{-1} \mathrm{~cm}^{-1}$ in asgrown samples, decreases considerably upon annealing in air. Reductive annealing in $\mathrm{Ar}: \mathrm{H}_{2}$ improves their conductivity. These films, without any post-growth annealing, are quite polycrystalline and ferromagnetic at $300 \mathrm{~K}$. The resistivity of all Co-doped ITO films exhibits thermally activated behavior. This is shown for films of two different compositions, which have been subjected to various thermal treatments, in Fig. 1. In high-conductivity samples the variation of the resistivity between 5 and $300 \mathrm{~K}$ is less than $5 \%$.

In Fig. 2, the XRD patterns of $\left(\operatorname{In}_{0.94} \mathrm{Sn}_{0.06}\right)_{2(1-x)} \mathrm{Co}_{2 x} \mathrm{O}_{3}$ films with $x=0.11$ (as-grown) and $x=0.3$ (annealed in air for $3 \mathrm{~h}$ at $400{ }^{\circ} \mathrm{C}$ ), as well as a pattern for the substrate, are shown. These patterns can be indexed as a cubic bixbyite structure of $\operatorname{In}_{2} \mathrm{O}_{3}$. The lattice constant shrinks with increasing Co content as shown in the inset of Fig. 2(a). This suggests that Co ions enter substitutionally into the ITO lattice. We observe no peaks in the XRD spectra corresponding to Co oxides or to metallic Co. Indeed, XPS spectra for films with $x \leqq 0.20$ show that the Co ions are in a high-spin divalent ground state-i.e., $\mathrm{Co}^{+2}\left(3 d^{7}, S=3 / 2\right)$. The Co $2 p$ corelevel spectra for $x=0.12$ (as-grown film) and for $x=0.18$ (film annealed for $3 \mathrm{~h}$ at $400{ }^{\circ} \mathrm{C}$ in air), shown in Fig. 3, exhibit a main Co $2 p_{3 / 2}$ peak at $780.05 \mathrm{eV}$ and the Co $2 p_{3 / 2}$ peak at $795.6 \mathrm{eV}(\Delta E=15.5 \mathrm{eV})$ which corresponds to a high-spin $\mathrm{Co}^{2+}$ state. ${ }^{13}$ The spectra shown were obtained after several Ar-ion etching cycles. The spectral line shapes and peak binding energies do not vary with successive etchings for these films. However, for $x \geqslant 0.20$, an additional signal appears in the XPS spectra after 10 min sputtering with $\mathrm{Ar}$ ions, with $\mathrm{Co} 2 p_{3 / 2}$ at $778.2 \mathrm{eV}$ and a Co $2 p_{3 / 2}$

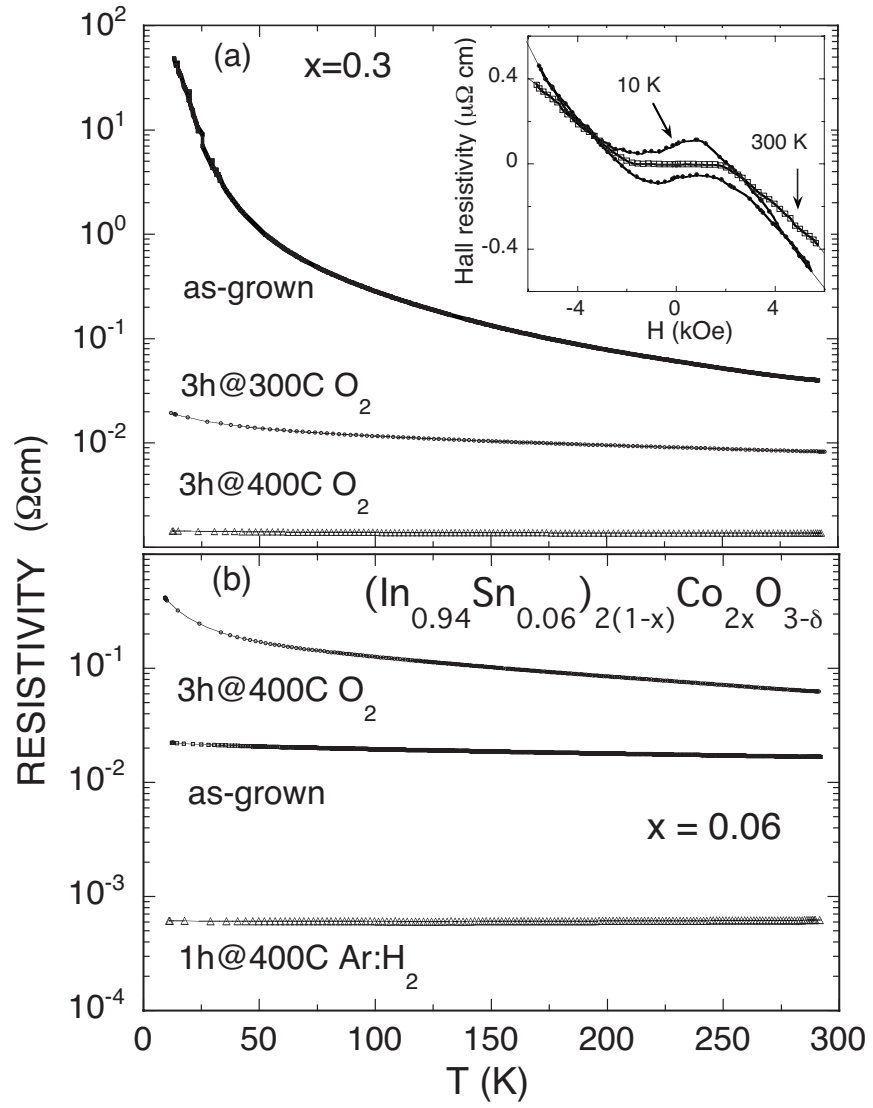

FIG. 1. Resistivity vs temperature for (a) $\mathrm{In}_{2} \mathrm{O}_{3}$ with $10 \mathrm{wt} \%$ $\mathrm{SnO}_{2}$ (ITO) films doped with 12 at. $\%$ of $\mathrm{Co}$, and (b) ITO films with 4 at. $\%$ of $\mathrm{Co}$, subjected to various thermal treatments. The inset in (a) shows magnetic field variation of the Hall resistivity in 12 at. \% of Co film annealed at $400{ }^{\circ} \mathrm{C}$ for $3 \mathrm{~h}$ in air.

$-2 p_{1 / 2}$ separation $\Delta E=15.0 \mathrm{eV}$, close to the one of metallic Co. ${ }^{13}$ These additional features are pointed to by arrows in Fig. 3. We find that a substantial fraction of the total Co atoms segregates to metallic Co clusters in this film at about $100 \mathrm{~nm}$ below the surface. Metallic Co clusters may form as a consequence of Ar-ion bombardment which can reduce oxidized Co ions and/or locally heat the sample and thus promote Co migration and subsequent clustering. However, we do not observe such an effect in films with $x \leq 0.2$ after similar etching cycles. Therefore, it is quite unlikely that the treatment with Ar ions would induce the clustering of Co observed in films with more than 8 at. \% of Co. Ion bombardment may induce changes in stoichiometry at the surface as well. This preferential sputtering is more pronounced for components with low mass and binding energy. We do not have any evidence of preferential sputtering in our films once a superficial layer of few nanometers has been removed.

A plan-view TEM image for a 12 at. \% of Co sample, exhibited as an inset in Fig. 2(c), also shows a hint of inclusions with diameters $\$ 3 \mathrm{~nm}$. These can be Co-metal or Corich nanocluster inclusions. On the other hand, the crosssectional TEM image of the ITO film with 4 at. \% of Co, exhibited as the inset of Fig. 2(b), shows a microstructure that is uniform and no clustering of Co ions nor secondary phases. This is in agreement with the XPS results. There is 


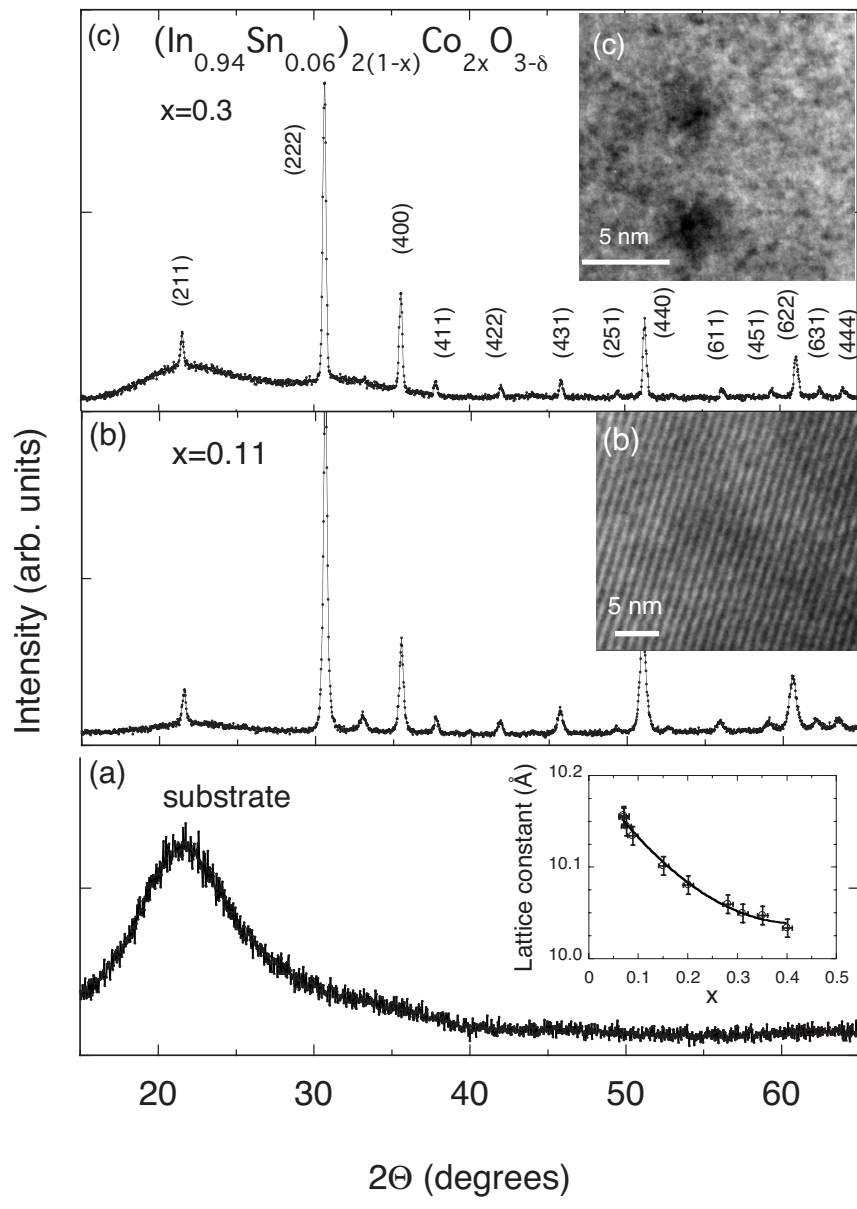

FIG. 2. X-ray diffraction patterns for (a) a fused-quartz substrate, (b) ITO as-grown film doped with 4 at. $\%$ of Co, and (c) ITO film with 12 at. $\%$ of $\mathrm{Co}$, annealed at $400{ }^{\circ} \mathrm{C}$ for $3 \mathrm{~h}$ in air, on a quartz substrate. The insets show a plan view transmission electron microscopy (TEM) image (c) and a cross-sectional TEM image (b) for the same samples. The inset in (a) exhibits variation of the lattice parameter with Co content in $\left(\mathrm{In}_{0.94} \mathrm{Sn}_{0.06}\right)_{2(1-x)} \mathrm{Co}_{2 x} \mathrm{O}_{3}$ films.

no significant variation in Co content with film depth, as probed by XPS. It seems that films with less than 8 at. \% of Co are well substituted. In films with higher Co content, Co metallic inclusions, in addition to the Co that enters substitutionally into ITO matrix, are quite likely. As discussed briefly in Sec. I, thermal annealing may affect the distribution of dopants in the host matrix. We do not observe any appreciable annealing effect on clustering of Co atoms up to $400{ }^{\circ} \mathrm{C}$. However, higher annealing temperatures might drastically change the microstructure of films as has been found in the $\mathrm{TiO}_{2}$ : Co system. ${ }^{9,10,14}$ Additional research on this point is underway.

Magnetic measurement results support the conclusions above. The zero-field-cooled and field-cooled susceptibilities are different for $T \leqq 120 \mathrm{~K}$ in films with more than 8 at. \% of Co. In addition, these films show hysteretic magnetization below room temperature and an anomalous Hall effect (AHE) ${ }^{15}$ How the Hall resistivity varies with external magnetic field is shown for one of $x=0.3$ films in the inset of Fig. 1(a). The AHE follows the magnetization behavior, as expected for a ferromagnetic semiconductor with spin-

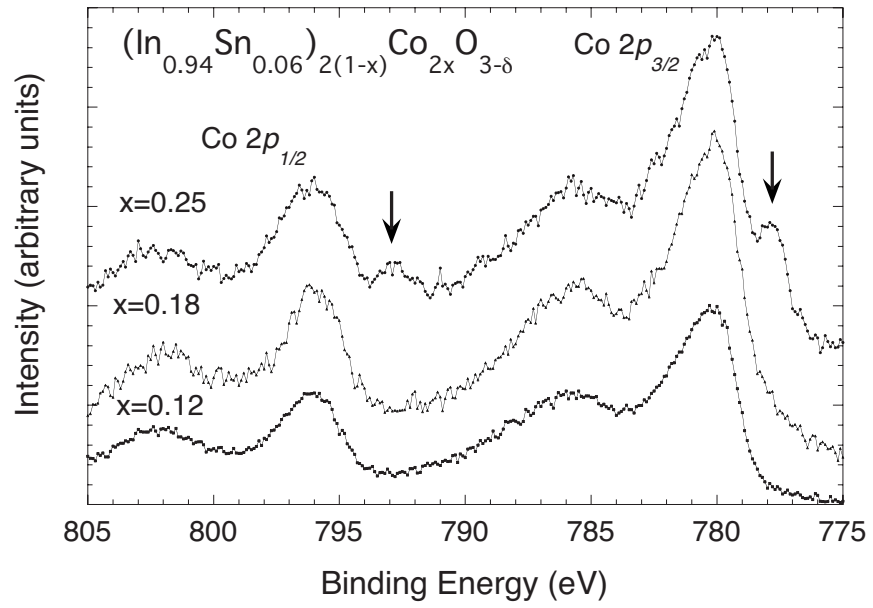

FIG. 3. Co $2 p$ core-level $\mathrm{x}$-ray photoemission spectra after several cycles of $\mathrm{Ar}^{+}$etching at $300 \mathrm{~K}$ for $\left(\mathrm{In}_{0.94} \mathrm{Sn}_{0.06}\right)_{2(1-x)} \mathrm{Co}_{2 x} \mathrm{O}_{3}$ samples with $x=0.12$ (as-grown), $x=0.18$ (annealed for $3 \mathrm{~h}$ at $400{ }^{\circ} \mathrm{C}$ in air), and $x=0.25$ (annealed for $3 \mathrm{~h}$ at $400{ }^{\circ} \mathrm{C}$ in air).

polarized carriers. Nevertheless, it has been found that $\mathrm{TiO}_{2}$ films with superparamagnetic Co clusters also show an AHE. ${ }^{14}$ Thus, the AHE cannot be used as a decisive criterion for intrinsic ferromagnetism. An assumption of two magnetic subsystems, interacting nanoclusters (of Co-metal or Co-rich ITO) and a ferromagnetic matrix, can account for the observed magnetic behavior in heavily doped ITO films. ${ }^{15}$ Further support for this follows from the field dependence of the magnetization shown in Fig. 4 for various Co concentrations. The diamagnetic background contribution from the substrate has been subtracted from all of the shown data. Films with $x \gtrsim 0.2$ show rather high $\mathrm{RT}$ values of the magnetic moment per Co ion $\left(\sim 1.7 \mu_{B}\right)$. These values are close to the ones for pure metal clusters $\left(1.67 \mu_{B} / \mathrm{Co}\right)$ and of Co nanoclusters $\left(2.1 \mu_{B} / \mathrm{Co}\right)$. Much smaller RT magnetic moments obtain for

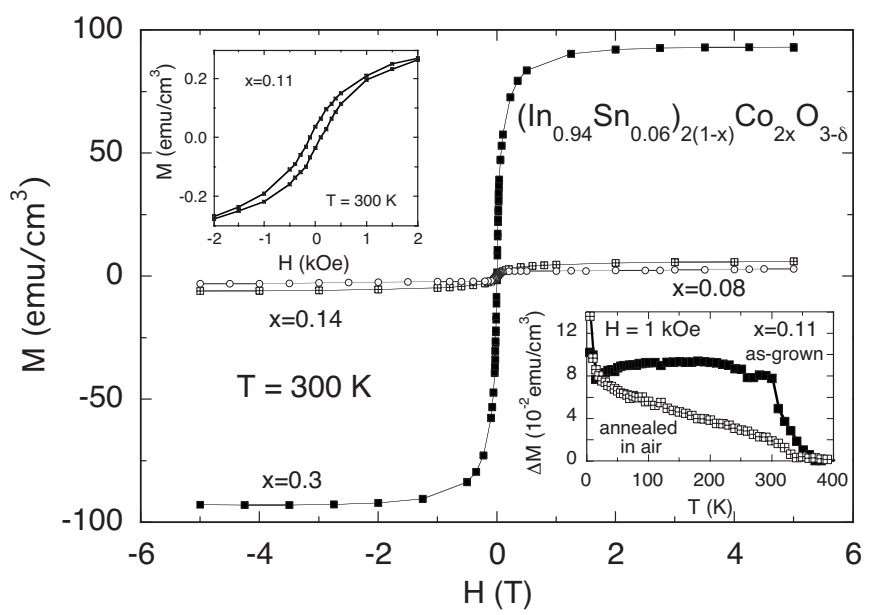

FIG. 4. Magnetization loops at $300 \mathrm{~K}$ for $\left(\mathrm{In}_{0.94} \mathrm{Sn}_{0.06}\right)_{2(1-x)} \mathrm{Co}_{2 x} \mathrm{O}_{3}$ with field applied parallel to the film plane. The lower inset shows the difference between field-cooled and zero-field-cooled magnetization measured at $1 \mathrm{kOe}$ for two ITO films with 4 at. \% of Co versus temperature. The upper inset shows a magnetic hysteresis loop at $300 \mathrm{~K}$ for the as-grown film with $x$ $=0.11$. 


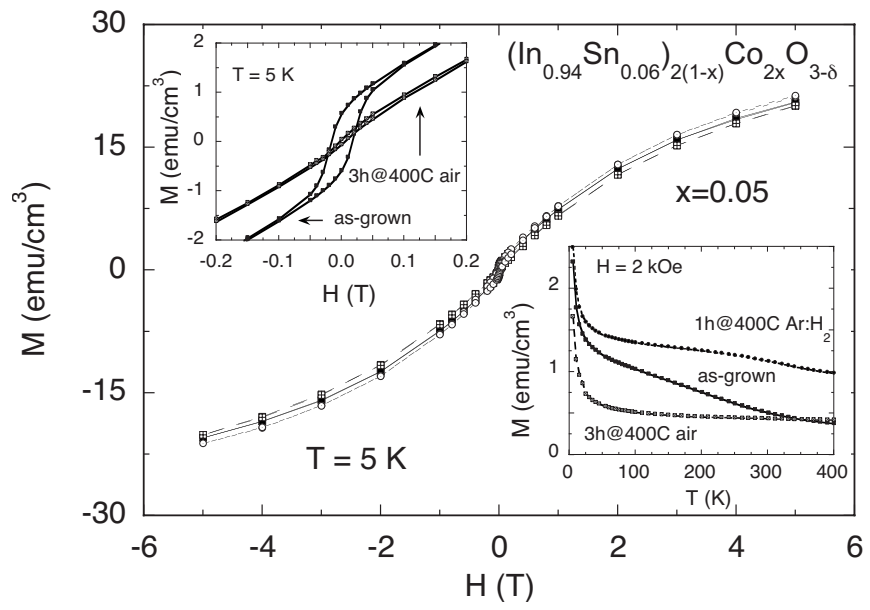

FIG. 5. Magnetization versus magnetic field at $5 \mathrm{~K}$ for $\left(\mathrm{In}_{0.94} \mathrm{Sn}_{0.06}\right)_{1.9} \mathrm{Co}_{0.1} \mathrm{O}_{3}$ films after various thermal treatments. The lower inset exhibits the temperature variations of magnetization for the same films. The upper inset shows the low-field $M-H$ curves for the as-grown film and the film annealed in air for $3 \mathrm{~h}$ at $400{ }^{\circ} \mathrm{C}$. The solid lines are to guide the eye.

homogeneous ferromagnetic ITO:Co films with less than 8 at. \% of Co $(x \leqslant 0.2)$. The ferromagnetic transition temperature $T_{c}$ increases with increasing Co content in this concentration range. It exceeds $400 \mathrm{~K}$ in $x=0.15$ films.

We now turn to the results obtained for films with less than 8 at. \% of Co. In the lower inset of Fig. 4, we show how the difference between field-cooled and zero-field-cooled magnetization (measured in a field of $1 \mathrm{kOe}$, applied parallel to the film plane) varies with temperature for two (as-grown and annealed in air for $3 \mathrm{~h}$ at $\left.400{ }^{\circ} \mathrm{C}\right) x=0.11$ films. A remnant of a ferromagnetic contribution is clearly present, even above room temperature, in both films. The upper inset shows the RT magnetic hysteresis loop for the same sample. Coercive fields of up to 100 Oe are observed. We find that the critical temperature $T_{c}$ and the average magnetic moment per dopant ion vary considerably with donor concentration and with the microstructure of these films. Annealing at temperatures higher than $600{ }^{\circ} \mathrm{C}$ gives rise to insulating and paramagnetic films.

The magnetization $M$ we measure in films with $x \leq 0.2$ is well below the saturation value $M_{s}$. Figure 5 shows the field dependences of magnetization $M(H)$ at $5 \mathrm{~K}$ for three films with 2 at. \% of Co: as-grown, annealed for $3 \mathrm{~h}$ at $400{ }^{\circ} \mathrm{C}$ in air, and treated for $1 \mathrm{~h}$ at $400{ }^{\circ} \mathrm{C}$ in $\mathrm{Ar}: 4 \% \mathrm{H}_{2}$. Their behavior at high fields is very similar although the low-field one is quite different (see the upper inset in Fig. 5). At this temperature only about $40 \%$ of the estimated saturation value is achieved at the highest applied field of $5 \mathrm{~T}$. There is a slowly saturating part, superimposed on FM magnetization, which clearly indicates that a large fraction of the $\mathrm{Co}^{+2}(S=3 / 2)$ magnetic moments do not contribute to the FM-ordered region. In order to estimate the magnetic moment per ferromagnetic Co ion, we approximate the isolated (paramagnetic) moments contribution $M^{\prime}$ to the total magnetization by a spin-only (no orbital contribution) $B_{3 / 2}(\alpha)$ Brillouin function with an argument of $\alpha=g \mu_{B} H / k_{B} T_{e f f}, M^{\prime} / M_{S}^{\prime}=B_{3 / 2}(\alpha)$. Here, the effective temperature $T_{e f f}=T+T_{A F}$ has an empirical
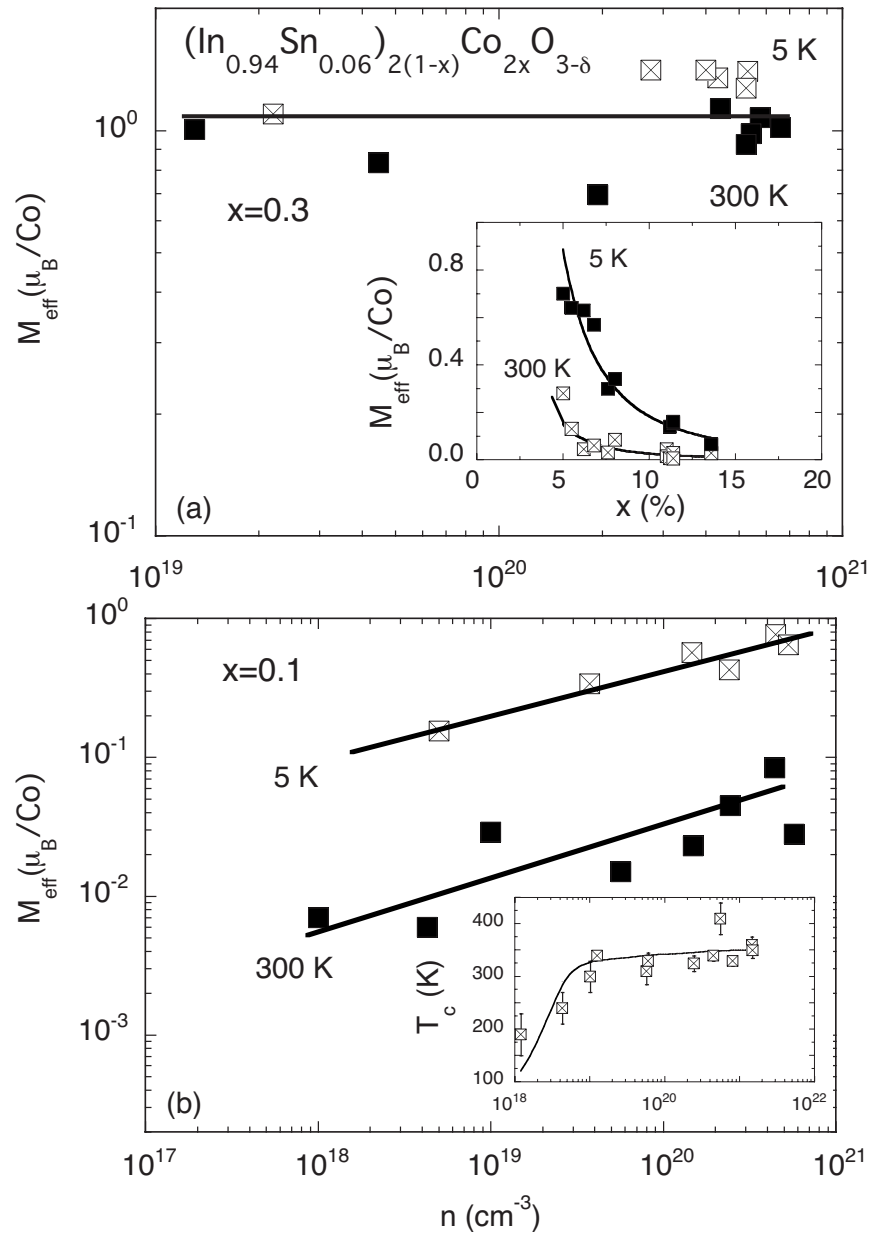

FIG. 6. Plots of magnetic moment per Co cation vs electron concentration at 5 and $300 \mathrm{~K}$ for (a) ITO films with 12 at. \% of Co and (b) ITO films with 4 at. \% of Co. The inset in (a) shows plot of the magnetic moment per Co cation vs Co content at 5 and $300 \mathrm{~K}$, for films with nearly equal electron concentration. The inset in (b) is a plot of the critical temperature $T_{c}$ vs RT electron concentration in ITO films with 4 at. $\%$ of Co. The solid lines are to guide the eye.

parameter $T_{A F}$ which describes the antiferromagnetic interaction between Co ions. We neglect the effect of small Co "clusters" (two to three atoms) which have a much smaller average magnetic moment than the isolated atoms. We find that more than the half of $\mathrm{Co}$ ion are paramagnetic in the films shown in Fig. 5. Estimated values of the magnetic moment per ferromagnetic Co atom, $M_{\text {eff }}$, obtained by subtracting the contribution of the paramagnetic Co ions from the total magnetization, are displayed in the inset of Fig. 6(a) versus Co content for films with approximately equal electron concentration. The observed decrease of the average moment per cation with increasing $x$ is likely brought about by antiferromagnetically coupled small clusters of Co cations. Pairs and groups of four Co atoms make no contribution while triplets contribute only $\mathrm{m} / 3$ to the total magnetization. Fitting a Curie-Weiss law $[m / H=C /(T-\Theta)]$ to the susceptibility data, shown in the lower inset of Fig. 5, yields small negative values for the Curie-Weiss temperature $\Theta$ $(\simeq-2 \mathrm{~K})$ in films with $x=0.05$. Similar values obtain for other compositions. This suggests weak antiferromagnetic 
interactions between free Co ions. In our fittings we use $T_{A F}=\Theta$.

Paramagnetic behavior is usually found in DMSs for doping levels lower than the site-percolation threshold number. A ferromagnetic behavior in ITO:Co films with low dopant concentration is surprising. Several observations are in order. First: thermal annealing at moderate temperatures (up to $300{ }^{\circ} \mathrm{C}$ ) in air or reducing atmosphere enhances ferromagnetism while higher annealing temperatures in air destroy it. As we grow our films with no oxygen pressure during deposition, the number of oxygen vacancies in as-grown films is large. These vacancies provide electrons to the conduction band. Tin atoms are not effective as dopants in as-grown ITO films which are amorphous. ${ }^{16}$ Most likely, Sn atoms occupy interstitial positions and some of them may form defects such as $\mathrm{SnO}_{x}$ which act as carrier traps rather than electron donors. Thermal annealing promotes Sn diffusion into In cation sites and, consequently, a rise in electron concentration. In addition, annealing promotes grain growth which leads to a decrease of donor sites trapped at the dislocations and grain boundaries. An enhancement of the magnetic moment at this stage strongly suggests a carrier-mediated exchange as the source of the observed intrinsic ferromagnetic ordering. Annealing at higher temperatures in air leads to compensation of substitutional $\mathrm{Sn}$ atoms by interstitial oxygen and to a decrease in the number of oxygen vacancies. ${ }^{16}$ Therefore, a deterioration of ferromagnetic coupling follows. The variations of $M(H)$ for the 2 at. \% films, shown in the upper inset of Fig. 5, agree with this model. We observe hysteresis for the as-grown sample [with $n(5 \mathrm{~K})=4.3 \times 10^{19} \mathrm{~cm}^{-3}$ ] while there is almost no FM signal for the same film treated in air at $400{ }^{\circ} \mathrm{C}$ for $3 \mathrm{~h}$ [with $n(5 \mathrm{~K})=1 \times 10^{18} \mathrm{~cm}^{-3}$ ]. Annealing in reducing atmosphere enhances FM contribution (not shown) and electron concentration.

Figure 6 shows how the magnetic moment per Co atom varies with total electron concentration $n$, obtained from high-field Hall resistivity $\rho_{x y}$ measurements. $\rho_{x y}$ at high fields shows a negative slope [see the inset of Fig. 1(a)] which is proportional to the inverse of $n$. We change the electron concentration in our films by varying the temperature and/or ambient gas while annealing. Interestingly, the magnetic moment of the ITO films doped with 4 at. \% of Co seems to correlate with the total electron concentration, both at 300 and $5 \mathrm{~K}$. (Note that the data cover more than three orders of magnitude in $n$.) The critical temperature $T_{c}$, displayed in the inset of Fig. 6(b), shows first a sharp rise with $n$ but varies more slowly for higher electron concentration. There is a large scatter in data points which arises mainly from our rough estimation of the effective magnetic moment. Nevertheless, the overall trend of an increasing $M_{\text {eff }}$ with increasing $n$ is clear and agrees with annealing effects. On the other hand, we note that there is no correlation between values of the magnetic moment and the electron concentration in the ITO films doped with 12 at. \% of Co [see Fig. 6(a)]. As discussed above, a mixed magnetic state of the films doped with more than 8 at. \% of Co may well obscures any correlation.

The observed correlation between $M_{\text {eff }}$ and $n$ strongly suggests a carrier-mediated exchange as a source of the intrinsic ferromagnetic ordering. Since electrons in the films are mostly localized, a bound magnetic polaron percolation model may be applicable. ${ }^{11,17}$ In this model, FM exchange is mediated by shallow donor electrons that form bound magnetic polarons. Overlap of polarons above some critical density of donors creates a spin-split impurity band. Such a scenario is favored in materials with a high dielectric constant and large density of donor defects. ITO:Co satisfies these requirements as the value of its high-frequency dielectric constant is $9,{ }^{12}$ and oxygen vacancies or tin dopants can be introduced in large numbers. Following Ref. 11, we estimate the percolation threshold for the appearance of long-range ferromagnetism in ITO:Co films as $\sim(0.5-1)$ $\times 10^{18}$ donors $/ \mathrm{cm}^{3}$ for the $\mathrm{Sn}$ atoms or oxygen vacancies, respectively. This agrees reasonably well with our experimental results. The films with $n \lesssim 1 \times 10^{18} \mathrm{~cm}^{-3}$ do not show ferromagnetic behavior. The percolation model provides a possible explanation for the low values of the magnetic moment we observe in films with $x \leqq 0.2$ as well. A large number of Co moments would not belong to the cluster of percolating bound magnetic polarons, which we expect to trigger ferromagnetic ordering and would therefore not contribute to the spontaneous magnetization. A direct Co-Co antiferromagnetic coupling provides an alternative mechanism, which would suppress both the critical temperature and the magnetization. We expect this coupling to be small since Co-Co separation is rather large for this composition.

\section{CONCLUSIONS}

We have grown indium-tin oxide thin films doped with Co by magnetron cosputtering at a deposition temperature $200{ }^{\circ} \mathrm{C}$. Their magnetic and electrical transport properties can be varied in a wide range by post-growth annealing in an oxidizing, inert or reducing atmosphere. ITO films with up to 8 at. \% of Co seem to be well substituted and show intrinsic FM behavior even above $400 \mathrm{~K}$. The effective magnetic moment of these films correlates with electron (donor) concentration. Our results point to a major role of oxygen vacancies in establishing ferromagnetic coupling in these materials. There is no clear correlation between the values of the magnetic moment and the number of oxygen vacancies in the ITO films doped with more than 8 at. \% of Co. Metallic inclusions, in addition to the Co that enters substitutionally into ITO matrix, are quite likely in these films which show mixed magnetic behavior.

\section{ACKNOWLEDGMENTS}

We acknowledge support from Ministerio de Ciencia y Tecnología of Spain through Grant No. MAT2005/1272. We thank Carmen Cosculluela and María Pilar Lozano for their valuable help in growing and characterizing films. 
*jolanta@unizar.es

${ }^{1}$ R. Janisch, P. Gopal, and N. A. Spaldin, J. Phys.: Condens. Matter 17, R657 (2005).

${ }^{2}$ P. Sharma, A. Gupta, K. V. Rao, F. J. Owens, R. Sharma, R. Ahuja, J. M. Osorio Guillen, B. Johansson, and G. A. Gehring, Nat. Mater. 2, 673 (2003).

${ }^{3}$ Y. Matsumoto, M. Murakami, T. Shono, T. Hasegawa, T. Fukumura, M. Kawasaki, P. Ahmet, T. Chikyow, S. Koshihara, and H. Koinuma, Science 291, 854 (2001).

${ }^{4}$ Z. Wang, W. Wang, J. Tang, L. D. Tung, L. Spinu, and W. Zhou, Appl. Phys. Lett. 83, 518 (2003).

${ }^{5}$ J. Philip, N. Theodoropoulou, G. Berera, J. S. Moodera, and B. Satpati, Appl. Phys. Lett. 85, 777 (2004).

${ }^{6}$ J. Philip, A. Punnoose, B. I. Kim, K. M. Reddy, S. Layne, J. O. Holmes, B. Satpati, P. R. Leclair, T. S. Santos, and J. S. Moodera, Nat. Mater. 5, 298 (2006).

${ }^{7}$ H. S. Kim, S. H. Ji, H. Kim, S-K. Hong, D. Kim, Y. E. Ihm, and W. K. Choo, Solid State Commun. 137, 41 (2006).

${ }^{8}$ N. H. Hong, J. Sakai, N. T. Huong, and V. Brizé, Appl. Phys. Lett. 87, 102505 (2005).

${ }^{9}$ S. R. Shinde, S. B. Ogale, S. DasSarma, J. R. Simpson, H. D. Drew, S. E. Lofland, C. Lanci, J. P. Buban, N. D. Browning, V.
N. Kulkarni, J. Higgins, R. P. Sharma, R. L. Greene, and T. Venkatesan, Phys. Rev. B 67, 115211 (2003).

${ }^{10}$ D. H. Kim, J. S. Yang, Y. S. Kim, T. W. Noh, S. D. Bu, S.-I. Baik, Y.-W. Kim, Y. D. Park, S. J. Pearton, J.-Y. Kim, J.-H. Park, H.-J. Lin, C. T. Chen, and Y. J. Song, Phys. Rev. B 71, 014440 (2005).

${ }^{11}$ J. M. D. Coey, M. Venkatesan, and C. B. Fitzgerald, Nat. Mater. 4, 173 (2005).

${ }^{12}$ I. Hamberg and C. G. Granqvist, J. Appl. Phys. 60, R123 (1986).

${ }^{13}$ Handbook of X-ray Photoelectron Spectroscopy, edited by G. E. Muilenberg (Perkin-Elmer Corporation, Eden Prairie, 1979), p. 78.

${ }^{14}$ S. R. Shinde, S. B. Ogale, J. S. Higgins, H. Zheng, A. J. Millis, V. N. Kulkarni, R. Ramesh, R. L. Greene, and T. Venkatesan, Phys. Rev. Lett. 92, 166601 (2004).

${ }^{15}$ J. Stankiewicz, F. Villuendas, J. Bartolomé, and J. Sesé, J. Magn. Magn. Mater. 310, 2084 (2007).

${ }^{16}$ J. R. Bellingham, W. A. Phillips, and C. J. Adkins, J. Phys.: Condens. Matter 2, 6207 (1990).

${ }^{17}$ S. Das Sarma, E. H. Hwang, and A. Kaminski, Phys. Rev. B 67, 155201 (2003). 